\title{
A case report of long term bevacizumab treatment in multiresistant ovarian cancer
}

\author{
Anette Kargo, Parvin Adimi, Karina Dahl Steffensen
}

Department of Oncology, Hospital Lillebaelt, Vejle, Denmark

Received February 08, 2016; Revised June 01, 2016; Accepted June 02, 2016; Published Online June 19, 2016

\section{Case Report}

\begin{abstract}
Treatment of multiresistant ovarian cancer is palliative and patients have needs for less toxic treatment. Anti-angiogenic treatments have a less toxic profile, and bevacizumab has shown improvement of progression free survival (PFS) in front-line trials. Bevacizumab is generally introduced in combination with chemotherapy; however this case report will describe the use of single-agent bevacizumab for more than five years (102 cycles) in a patient with relapse of advanced ovarian cancer.
\end{abstract}

Keywords: Ovarian cancer; Bevacizumab

\section{Introduction}

In Denmark 500 new cases of ovarian cancer (OC) are registered yearly. Approximately $75 \%$ of the women have advanced disease at the time of diagnosis (stage II-IV) and around $70-85 \%$ will have initial response to treatment. Nevertheless $80 \%$ will experience relapse, often within 2 years after first-line chemotherapy, and the chance of cure is small.1,2 Over time patients develop chemotherapy resistance and there is an obvious need for biological treatment without hematological toxicity side effects. Biological treatment regimens, including anti-angiogenic therapy have the advantage of rare hematological toxicity.

Bevacizumab treatments have been investigated in several studies and have shown improvement of PFS in both first and second/third line treatment although it is probably with more striking effect in patients with recurrence. ${ }^{3}$ Bevacizumab has been approved in Europe for first-line treatment when administered in addition to carboplatin and paclitaxel and as maintenance therapy after completion of the chemotherapy based on two pivotal phase III studies, ICON7 and GOG218.4,5,6

Two further studies in ovarian cancer patients with recurrent cancer have also been published.7,8 The first study (OCEANS) included patients with platinum-sensitive disease, while the second study (AURELIA) was in platinum-resistant disease. These studies looked at the effect of adding bevacizumab to a combination regime with carboplatin and gemcitabine or to paclitaxel, topotecan, or pegylated liposomal doxorubicin and both studies indicated an improved PFS in patients treated with the addition of bevacizumab to chemotherapy. The hallmark of these large studies and the approved indication is the combination with chemotherapy. Since patients with recurrence of disease often have a poorer performance status and different requirements for quality of life in palliation of a life-threatening disease, it is highly relevant to examine treatments with less side-effects including the possibility of single-agent bevacizumab treatment which is much less well documented.

\section{Case Presentation}

A 55-year-old post-menopausal woman debuted in 2004 with pleural effusion. Pleural effusion cytology was investigated by immonohistochemistry. Abdominal ultrasound found discreet amount of ascites without any ovarian pathology described. Physical exam was normal. Furthermore, computed tomography of thorax, abdomen and pelvis were without any sign of primary tumor. Since it was not possible to detect the primary tumor the patient was considered to have lung cancer and treated with standard platinum and vinorelbine. After the first dose the patient developed febrile neutropenia, and the second dose was reduced. Despite dose reduction the patient developed pancytopenia and subsequent cycles were delayed several times. After 6 cycles, a CT scan showed that the patient had complete remission and the patient entered the follow-up program. In February 2007 the patient discovered lower abdominal fullness, and was diagnosed with grade 3 , mixed serous/clear cell

Corresponding author: Anette Stolberg Kargo; Department of Oncology, Hospital Lillebaelt, Vejle, Denmark

Cite this article as: Kargo A, Adimi P, Steffensen $\mathrm{K}$. A case report of long term bevacizumab treatment in multiresistant ovarian cancer. Int J Cancer Ther Oncol. 2016; 4(2):4214. DOI: 10.14319/ijcto.42.14 
OC, stage IIIC. She underwent surgery with total hysterectomy, bilateral salpingo-oophorectomy and omentectomy. The surgery was not complete as a $3 \mathrm{~cm}$ large tumor nodule was left above the liver. A pathology reexamination of the previous pleural effusion and present tumor was performed and showed that the tissues had the same morphology, concluding that the patient now had relapse from a previous OC.

Post-operatively, the patient received first-line treatment carboplatin and paclitaxel at a reduced dose due to the previous incidence of pancytopenia. Paclitaxel was withdrawn after the second dose due to hematological toxicity. The patient continued carboplatin monotherapy, and despite supplementary filgrastim the treatment was delayed due to pancytopenia. After 4 doses of carboplatin the treatment was changed to gemcitabine, but since the patient again developed pancytopenia she was referred to follow-up. In June 2008, after a treatment-free interval of 8.8 months the patient was diagnosed with progression and liver metastases and referred to experimental treatment with single-agent bevacizumab in the hope for better tolerance.

In July 2008 single-agent bevacizumab treatment was initiated (10 mg/kg intravenous infusion) on day 1 of a 21 days cycle. Serum cancer-related antigen 125 (CA125) at baseline was $49 \mathrm{U} / \mathrm{mL}$ (normal range $0-35$ ). After 3 doses of bevacizumab CA125 dropped to $8 \mathrm{U} / \mathrm{mL}$ and remained stable (range 8-11) until September 2012. After 4 years of treatment (70 doses) bevacizumab was paused because the patient needed surgery for mitral-valve-disease. In February 2013 after a 6 months' treatment pause, CA125 had increased to $290 \mathrm{U} / \mathrm{mL}$, and a CT scan verified progression. Bevacizumab treatment was reintroduced in March 2013, and CA125 dropped to $65 \mathrm{U} / \mathrm{mL}$ after the first 3 cycles of re-induction and the patient stayed on treatment for an additional 32 cycles with hardly any side effects (Figure 1). The patient tolerated the treatment without any significant toxicities and reported a good quality of life during treatment. In December 2014 after a total of 102 cycles of bevacizumab CA125 progressed to $231 \mathrm{U} / \mathrm{mL}$ and a CT scan confirmed progression, and third-line treatment with doxorubicin was introduced in February 2015 with CA125 response but with a new CA125 progression after 8 cycles of treatment.

\section{Discussion}

Knowledge about single-agent bevacizumab in women with recurrent OC is limited since most studies have evaluated bevacizumab in combination with standard chemotherapy regimens. Two trials ${ }^{7,8}$ on relapse treatment allowed single-agent bevacizumab until progression, $10 \mathrm{mg} / \mathrm{kg}$ every 2 weeks and $15 \mathrm{mg} / \mathrm{kg}$ every 3 weeks, respectively. However, only a few patients received long-term bevacizumab maintenance. The longest duration was 24 and 37 treatments, respectively.7,8 Bevacizumab single agent treatment for recurrent $O C$ is described in the literature with a dose of $15 \mathrm{mg} / \mathrm{kg}$ every 3 weeks with 16 and 35 cycles as the highest number of cycles reported.9,10 A single study describes long term effect with one patient having received treatment for a period of more than 21 months and still ongoing at the time of publication. ${ }^{10}$

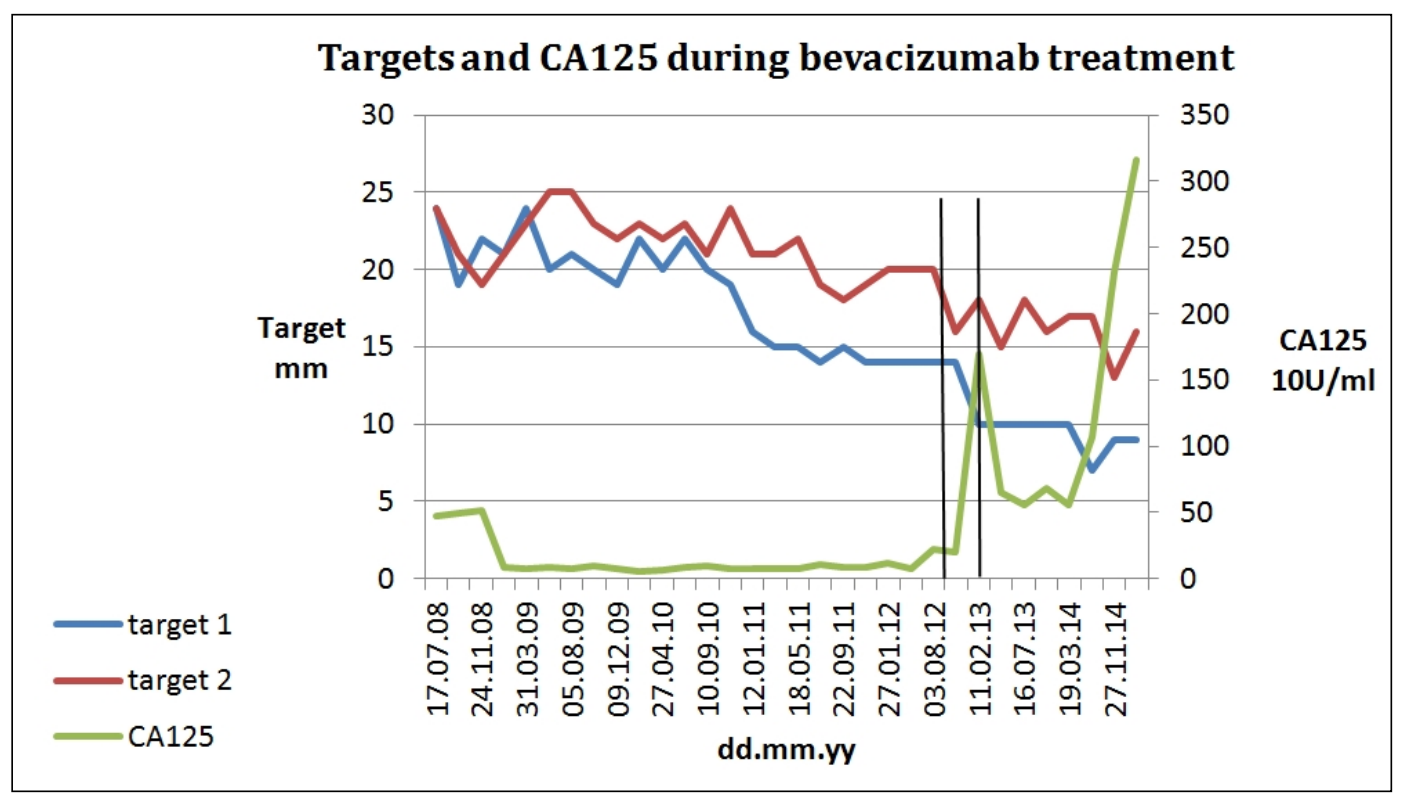

Figure 1: CA125 level during single treatment with bevacizumab, $10 \mathrm{mg} / \mathrm{kg}$ every 3 weeks. Treatment was paused for 6 months, during September 2012 until February 2013 - illustrated with the two vertical black lines. 
The patient in this case report was treated with single agent bevacizumab $10 \mathrm{mg} / \mathrm{kg}$ every three weeks for $51 / 2$ years. This treatment managed to keep the disease stable despite the lower dose compared to several published trials. The disease progressed during treatment pause, and when bevacizumab was reintroduced, the tumor responded again. This supports the assumption that the patient clearly has underlying cancer disease, which bevacizumab was able to keep stable. Our patient was initially treated with different chemotherapy regimens, all causing severe hematological toxicity and repeated treatment delays. During the treatment with bevacizumab there were no side-effects and the patient benefited from the treatment for several years.

It is our experience that patients with advanced OC can benefit from single-agent bevacizumab. This case was exceptional in keeping the cancer stable for more than 5 years, which has not previously been described in the literature.

\section{Conflict of interest}

The authors declare that they have no conflicts of interest. The authors alone are responsible for the content and writing of the paper.

\section{Acknowledgement}

The authors thank the patient who participated in this study.

\section{References}

1. Available from http://eu-cancer.iarc.fr/EUCAN/CancerOne.asp $\underline{x}$ ?Cancer $=27$ \&amp;Gender $=2$

2. Luvero D, Milani A, Ledermann JA. Treatment options in recurrent overaian cancer: latest evidence and clinical potential. Ther Adv Med Oncol. 2014;6(5): 229-39.
3. Monk BJ, Pujade-Lauraine E, Burger RA. Integrating bevacizumab into the management of epithelial ovarian cancer: the controversy of front-line versus recurrent disease. Ann Oncol. 2013;24(suppl.10):x53-8.

4. Available from

http://www.ema.europa.eu/docs/en_GB/docu ment library/Summary of opinion/human/00 0582/WC500112811.pdf

5. Burger RA, Brady MF, Bookman MA et al. Incorporation of Bevacizumab in the Primary Treatment of Ovarian Cancer. N Engl J Med. 2011;365:2473-83.

6. Perren TJ, Swart AM, Pfisterer J et al. A Phase 3 Trial of Bevacizumab in Ovarian Cancer. N Engl J Med. 2011;365:2484-96.

7. Pujade-Lauraine E, Hilpert F, Weber B et al. Bevacizumab Combined With Chemotherapy For Platinum-Resistant Recurrent Ovarian Cancer: The AURELIA Open-Label Randomized Phase III Trial. J Clin Oncol. 32:1302-8.

8. Aghajanian C, Blank SV, Goff BA et al. OCEANS: A Randomized, Double-Blind, Placebo-Controlled Phase III Trial of Chemotherapy With or Without Bevacizumab in Patients With Platinum-Sensitive Recurrent Epithelial Ovarian, Primary Peritoneal, or Fallopian Tube Cancer. J Clin Oncol. 2012;30:2039-45.

9. Cannistra SA, Matulonis UA, Penson RT et al. Phase II study of bevacizumab in patients with platinum-resistant ovarian cancer or peritoneal serous cancer. J Clin Oncol. 2007;25:5180-6.

10. Burger RA, Sill MW, Monk BJ et al. Phase II trial of bevacizumab in persistent or recurrent epithelial ovarian cancer or primary peritoneal cancer: a Gynecologic Oncology Group Study. J Clin Oncol 2007;25(33):5165-71. 